\title{
Market segmentation of wireless sensor system in network commodity selection
}

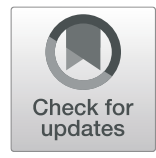

Fuxiang Liu

\begin{abstract}
Online product recommendation and market segmentation are directly related to the consumer experience and the healthy development of the e-commerce market. In order to improve the selection of network products and the effect of market segmentation, this study introduced a wireless sensing system based on the recommendation of traditional network products and combined the operational conditions of the network commodity market to construct a personalized recommendation e-commerce system. Meanwhile, a series of representative WSN congestion control strategies are designed by dynamically adjusting the network topology, routing algorithm, channel allocation protocol, and channel resource utilization, and the system structural components and functions are implemented. The research shows that the proposed algorithm and system have certain practical effects and can provide theoretical reference for subsequent related research.
\end{abstract}

Keywords: Wireless sensing system, Network commodity, Application, Market segmentation, Commodity selection

\section{Introduction}

Similar to shopping on e-commerce sites, many organizations are now launching online product recommendation services. Faced with a large number of online products, how to choose the most suitable product recommendation service for users is the key research direction of ecommerce development. In the e-commerce website, the user's interest and preferences are predicted by collecting and analyzing the user's purchase record, browsing history, and other related information, thereby recommending the products that he may be interested in [1]. Similarly, the user-specific intelligent push system proposed in this paper will focus on how to intelligently push suitable online commodity services for users. In this system, the user profile data collected in the Internet of Things and some other personal information of the user will be combined with the idea of data mining to analyze the health services and commodities required by the target users, and finally push the recommendation results to the user browsing interface.

In the e-commerce industry, providing products according to customer needs and enabling customers to easily find the products they want to buy is the key to gaining competitive advantage. The practice of foreign

Correspondence: liufms@ctgu.edu.cn

Science College and Three Gorges Mathematical Research Center, China Three Gorges University, Yichang 443002, China e-commerce enterprises shows that category management is a very effective management method for ecommerce enterprises to gain competitive advantage and increase market share. Although category management has only been born for 20 years, the effectiveness of category management has attracted the interest of many domestic and foreign scholars. Through continuous practice and research, many representative viewpoints have been proposed. Fader and Lodish [2] found that consumer characteristics such as the frequency of purchase have a certain explanatory power for the pricing and promotion environment of supermarket goods. Hoch [3] and others studied the relationship between the market price elasticity of various commodities and the demographic characteristics of customer groups. Raiu [4] studied the difference in sales of different types of goods and established its relationship with category characteristics and marketing groups and variables. The results of Poel [5] and others show that the complementarity between commodities is directly proportional to the interaction of promotions. These studies strongly support the rational identification of commodity classification theories based on consumer demand. Compared with European and American countries, China's research is later. In 1997, the Hong Kong Supply Chain Management Advisory Committee was established, and the 
category management experiment was carried out. Then, the Taiwan area was actively promoted. The category management in the mainland is promoted by the China ECR (Efficient Consumer Response) committee established in 2001. Zhang Hongxia and Zhang Songjie [6] first analyzed the significance, basic steps, and key factors of product category management in category management in 1999, and the application prospects of category management are promising. He Yun [7] discussed the optimization management of category, and he believes that the purpose of the optimization management of the real category is to make the shelf most valuable, and the product portfolio placed on the shelf is the customer's favorite product. Xia Weichao [8] deeply analyzed the relationship between satisfying customer needs and supplier management and believed that category management should be based on category accounting, which is designed to meet consumer needs and manage suppliers' new management methods. Wu Zikai [9] emphasized the importance of category tactics.

On the whole, the focus of the above research is different. It provides a reference for the research of the category management model from various angles, which is very helpful for the next step. In the enterprise management category management process, because there is no corresponding category management software system to help enterprises to manage the process, system, and actualization, the enterprise lacks effective information technology support, which leads to the time-consuming and unsatisfactory decision-making process of the category management decision-making process. The category management model system is a business information management system. From the perspective of developed countries, the business information management system is divided into six stages: automatic settlement management of cash registers, sales analysis management of POS machines, inventory management, storage and transportation management, integrated management, data warehousing, and data mining [10]. In other words, the computer information management system can be roughly divided into three levels: one is the POS system, the second is the point-of-sale sales management system, and the third is the decision support system [11]. At present, domestic supermarket enterprises have realized the coverage of POS machines and can monitor the sales situation, inventory situation, and storage and transportation situation of goods in real time, and can monitor and dynamically manage the quantity, location, and price of goods. That is to say, the computer management system of most supermarket retail enterprises still stays in the collection of basic data. However, with the improvement of marketing management level, the current point-to-point sales service of the system can no longer meet the needs of supermarket managers. Therefore, managers want the system to automatically analyze sales data, mine customer preferences, and provide decision support. However, in practice, enterprises lack confidence in information systems, and they believe that information systems cannot solve the practical problems of category management. As a result, companies are struggling to deploy a category management system. On the other hand, it can be seen in the enterprises that have already been implemented that based on the information management software currently used, most enterprises can collect basic internal data required for category management. However, the system has not been used in the analysis of data or does not have the ability to analyze data and report data required by category management [12].

In summary, current network products require a category management software that meets the actual characteristics. The software provides systematic and scientific services that improve the relationship between suppliers and retailers, improve customer satisfaction, reduce costs, and increase profits.

\section{Classical congestion algorithm of wireless sensor networks}

WSN is a wireless network with no data center, its nodes form a network through white organization and adaptive, and there is no control center. In order to improve the quality of network services and prolong the service life of the network, based on the above-mentioned causes of congestion caused by WSN, researchers around the world have designed a series of representative WSN congestion control strategies by dynamically adjusting network topology, routing algorithms, and introducing channel allocation protocols to improve channel resource utilization [13].

\subsection{CODA}

Congestion detection avoidance (CODA) also known as congestion prediction and avoidance algorithm, is a classic WSN congestion control scheme. The program is mainly divided into three major operational mechanisms (Fig. 1) [14].

The CODA algorithm timely realizes the prediction of network congestion, and achieves network congestion mitigation, avoidance, and cancellation by reducing the data transmission rate of the source node for a long time. However, during the running process, the upstream congestion node performs the local congestion control policy by using the AIMD policy (discarding the cached data packet). Moreover, it adjusts the transmission rate of the multi-source node that sends the data packet to the receiving end through back pressure adjustment, and the rate is different, which is not conducive to maintaining the WSN quality of service (QOS 


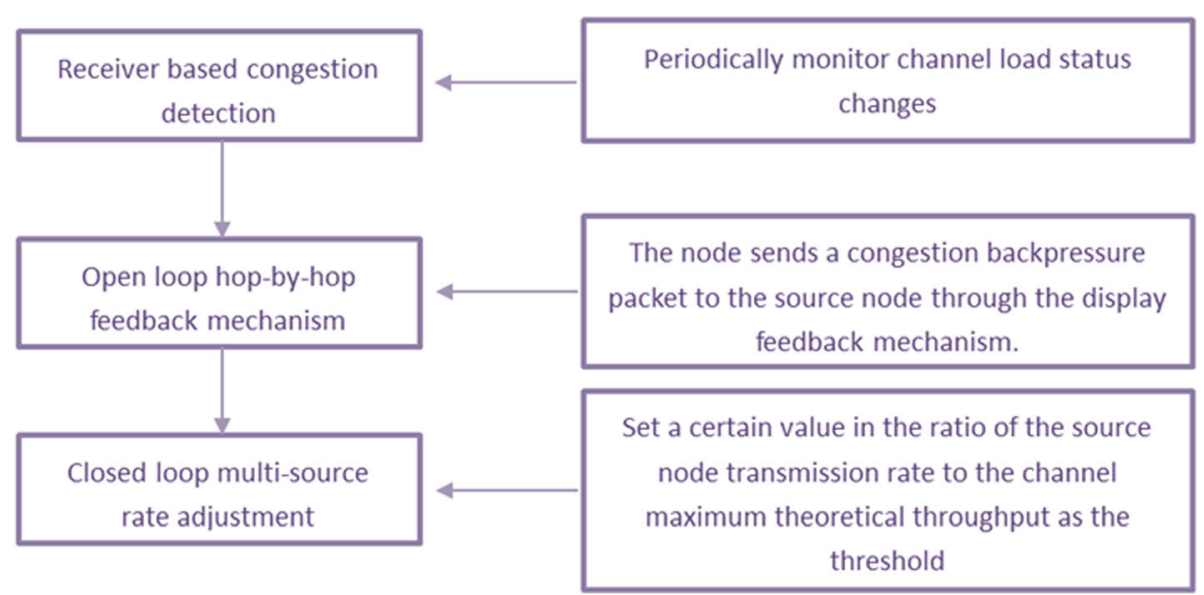

Fig. 1 Running Flow chart of the CODA algorithm

performance) and fairness, and the channel sensing also consumes a lot of energy [15].

\subsection{ESRT}

Event-to-sink reliable transport (ESRT) is a reliable event transmission protocols. The congestion control strategy of ESRT realizes the reliability of network data transmission and the effectiveness of congestion control by changing and transforming the network state. The sensor nodes and the aggregation nodes in the network directly transmit data. The ESRT acts on the SINK node to obtain the network congestion detection by adjusting the network transmission status to obtain the source node transmission rate, and the number of packets received by the aggregation node in each period determines the reliability of the network transmission. The ESRT congestion control policy operation relies on the following five network states: Reliability states $R=(\mathrm{LR}$, $\mathrm{HR})$, network congestion $C=(N C, C)$

$$
S=R^{T} \otimes C
$$

network states $S_{i} \in\{(\mathrm{LR}, \mathrm{NC}),(\mathrm{LR}, \mathrm{C}),(\mathrm{HR}, \mathrm{NC}),(\mathrm{HR}$, C), OOR\}, LR-low reliability, HR-high reliability, $\mathrm{NC}-$ no congestion, $\mathrm{C}-$ congestion, and OOR-best state. The ESRT strategy can guarantee the reliability of the network and the quality of network services. However, the SINK node communicates directly with the source node and does not support multi-hop clustering routing and topology mechanisms and is not suitable for large wireless sensor networks [16].

\subsection{Congestion control strategy of adaptive resource}

When detecting that the network is in a congested state, waking up the dormant node (increase network resources), changing the network topology, and forming a multi-emission route can alleviate the many-to-one data transmission mode, and achieve network congestion cancellation through multi-channel communication. After the congestion is released, the node without data transmission continues to sleep (reducing network resources), and the multi-route and multi-channel communication modes are released. The algorithm improves energy utilization, extends network lifetime, and ensures network stability and OOS performance.

\subsection{DCCP-data packet congestion control protocol}

The DCCP congestion control protocol establishes, maintains, and disassembles the reliable handshake negotiation mechanism at both ends and users can adopt different congestion control mechanisms according to different applications. Different congestion control policies are described by the congestion control flag (CCID), and CCID2 implements congestion mitigation and release by adopting a cache queue date packet of a drop node similar to the AIMD protocol. CCID3 implements congestion control by adjusting the transmission rate of source node data, which is similar to the TFRC protocol. However, the DCCP protocol cannot guarantee the high reliability of the network. The high bit error rate leads to an increase in network data packet loss rate and reduces the throughput of the DCCP congestion control protocol.

\subsection{An algorithm for avoiding node congestion in line sensor networks}

The strategy adopted by the algorithm is to allocate the congestion avoidance of the sending window to the source node and to set the priority for the data packet scheduling. The upstream node allocates a transmission window for the downstream node according to a certain policy, and the node sets the priority for the data packet in the cache queue, and the data packet with the highest priority is always transmitted in the effective transmission window. 
Although the algorithm effectively achieves congestion avoidance and improves energy utilization and improves the fairness of network transmission and reduces network transmission delay, it still lacks efficient congestion detection mechanism and time synchronization algorithm for end-to-end data transmission.

\subsection{COMUTI is a cluster-based data traffic congestion control algorithm}

During the operation of the COMUT, the node detects its own data transmission traffic and reports it to the cluster head node within a certain time interval. The cluster head node determines whether the local traffic is greater than the threshold according to the received data. If it is greater than the threshold, the AIMD mechanism is used to adjust the transmission rate of the upstream node to implement congestion control and release. This algorithm affects the stability of network throughput and improves data transmission delay [17].

Based on the specific application scenarios, these algorithms establish an efficient WSN congestion control strategy system for congestion detection, congestion avoidance, and congestion cancellation.

(1) Nodes close to the SINK node area are used as relay nodes for network-wide data transmission and become "hot spots" for power consumption and congestion. (2) Due to the limited bandwidth of nodes and the low level of endto-end communication links, network congestion may occur in some cluster head nodes or common nodes.

\section{Congestion control strategy based on CCBDC- RSUL algorithm}

This paper is a wireless sensor network model based on clustering topology. In the case of a sudden increase in data volume, it is easy to cause two phenomena of in-cluster and cluster head congestion and cluster head congestion in SINK area. For the problem of congestion in the intracluster, a sub-cluster head VCH (Vice Cluster Head) is selected in the non-cluster head node $\mathrm{CM}$ to share the data stream for the cluster head node $\mathrm{CH}$, and the congestion in the intra-cluster can be implemented. For the problem of inter-cluster congestion (SINK area congestion problem), the network area is non-uniformly layered, and the dormant nodes near the congested nodes are awakened and become cluster heads. Then, the two major strategies of the LEMPT optimized communication path implement intercluster congestion cancellation.

The network determines whether it is in a congested state by using periodic congestion detection. If congestion is not detected in the period, it enters congestion detection in the next network period. If a congestion trend is detected, the network determines whether the congestion is intra-cluster or inter-cluster by the size of the $P$. When $P \in(0,0.5)$ is detected, the network is in an intra-cluster congestion state, and the clustering-based data traffic congestion control algorithm (COMUT) algorithm is used in the intra-cluster to obtain the subcluster head (VCH). Moreover, the nodes in the intracluster choose which cluster head to join according to the minimum value of the communication link energy consumption and update the node route to clustered. After that, it is judged whether there is congestion of inter-cluster. If there is, it will enter the inter-cluster congestion control link, otherwise it will detect whether the congestion is released. If it is judged that the network is in a state of inter-cluster congestion, the network is first non-uniformly layered, and a LEMPT treelike optimization path is established between the clusters and the cluster head. Next, it is checked whether congestion has been alleviated or released. If the congestion is not alleviated, the node (CM) that is adjacent to the congestion cluster head $(\mathrm{CH})$ and has the highest energy is awaked and become the cluster head, and the LEMPT is updated. After that, it is detected whether the congestion is released. If it is not released, it enters the congestion control loop again, and if congestion has been removed, the congestion control resource is deleted [18].

According to the congestion detection of the network by combining the queue length of the node buffer and the congestion degree, it can be seen that when $B_{p 1}<$ $B_{p} \leq B_{p 2} \cap(C(n)>1)$ or $B_{p}>B_{p 2}$, the network $\mathrm{CH}$ node will be congested, and the congestion control link needs to be entered. The congestion type is determined according to the ratio $P$ from the intra-cluster data traffic to the inter-cluster data traffic. When the data traffic from the cluster is much larger than the data traffic from the cluster, it is determined that the congestion belongs to intra-cluster congestion; when $0<P<0.5$, the data traffic from the inter-cluster is much larger than the data traffic from the inter-cluster, and the congestion is determined to belong to intra-cluster congestion. When $0.5 \leq P \leq 1.5$, the network is judged to be intra-cluster and inter-cluster congestion. In both cases, the network must first enter the congestion control phase in the intra-cluster.

We divide the nodes of the whole network into important, minor and general three levels according to their priorities. The areas where the node timers randomly select the set values are sequentially increased. The division of each area is shown in equation (1) [19]:

$$
T_{i} \in\left\{\begin{array}{l}
(0.8,1] t, i \in A \\
(1,1.4] t, i \in B \\
(1.4,2] t, i \in C
\end{array}\right.
$$

In the formula, $t$ represents the average value set by the previous time, $A$ is a set of important nodes, $B$ is a set of secondary nodes, and $C$ is a set of general nodes. 
According to the principle of formula (1), the set value is randomly selected for each node timer, and if the node data times out in the node cache queue, the node becomes a $\mathrm{VCH}$.

In the clustering process of nodes, the coordinates of $\mathrm{CH}$ are $\left(x_{\mathrm{ch}}, y_{\mathrm{ch}}\right)$, the coordinates of $\mathrm{VCH}$ are $\left(x_{\mathrm{vc}}\right.$, $\left.y_{\mathrm{vc}}\right)$, and the coordinates of CM are $\left(x_{i}, y_{i}\right)$. The principle of adding the corresponding cluster heads and clustering is shown in Eqs. (2), (3), and (4).

$$
\begin{aligned}
d_{i-\mathrm{ch}} & =\sqrt{\left(x_{i}-x_{\mathrm{ch}}\right)^{2}+\left(y_{i}-y_{\mathrm{ch}}\right)^{2}} \\
d_{i-\mathrm{vch}} & =\sqrt{\left(x_{i}-x_{\mathrm{vc}}\right)^{2}+\left(y_{i}-y_{\mathrm{vc}}\right)^{2}} \\
U_{i} & =\left\{\begin{array}{l}
U_{c h}, d_{i-\mathrm{ch}}<d_{i-\mathrm{vch}} \\
U_{\mathrm{vch}}, d_{i-\mathrm{vch}}<d_{i-\mathrm{ch}}
\end{array}\right.
\end{aligned}
$$

In formula (4), $U_{\mathrm{ch}}$ denotes a cluster set with $\mathrm{CH}$ as a cluster head, $U$ denotes a cluster set with $\mathrm{VCH}$ as a cluster head, and $U_{i}$ denotes a node element within the cluster. $d_{i \text {-ch }}$ and $d_{i \text {-vch }}$ respectively represent the distance between the nodes $\mathrm{CM}$ to $\mathrm{CH}$ and $\mathrm{VCH}$. The two cluster regions are segmented as shown in Fig. 2 [20].

First, the distances $d_{i-\mathrm{ch}}$ and $d_{i-\mathrm{vch}}$ of the nodes $i$ to $\mathrm{CH}$ and $\mathrm{VCH}$ in the cluster are respectively determined, and the sizes of the two are determined. Which of the distance values is small, then node $i$ is added to the cluster head. If the distance between the two is equal, the remaining energy of the two cluster heads is judged, and the node $i$ is added to the cluster head with the larger remaining energy.
When $\left(B_{p 1}<B_{p} \leq B_{p 2}\right) \cap(C(n)>1) \cap(P>1.5)$ or $B_{p}>$ $B_{p 2} \cap(P>1.5)$, network congestion belongs to intercluster congestion, and the network directly enters the inter-cluster congestion control phase.

When $\left(B_{p 1}<B_{p} \leq B_{p 2}\right) \cap(C(n)>1) \cap(0.5 \leq P \leq 1.5) \quad$ or $B_{p}>B_{p 2} \cap(0.5 \leq P \leq 1.5)$, network congestion belongs to cluster-to-cluster congestion.

After the network releases, the congestion within the cluster through intra-cluster congestion control, the network can enter the cluster congestion control phase (congestion mitigation and congestion cancellation).

Our work introduces the ULEE algorithm to achieve non-uniform stratification of the network, aiming to solve the "hot spot" problem near the SINK node area. Li Chengfa and others proposed the EEUC agreement, whose main ideas are: Since multi-hop communication between WSN clusters is used to realize data transmission between the cluster head and the SINK node and the nodes in the network are equivalent, the closer to the nodes in the SINK node area, the more data relay pressure is assumed. Furthermore, it is completed by introducing two major parameters of $\mathrm{C}$ and $R_{\text {cmax }}$.

For the non-uniform layered design of the network, the closer to the SINK node, the smaller the layer, the smaller the cluster size, and the fewer the number of nodes in the intra-cluster. By alleviating the load of data acquisition, processing, and transmission of nodes in the area, more energy and transmission resources are used for data relay transmission of the whole network, and congestion reduction is implemented by using UL (uneven layer). Formula (5) is

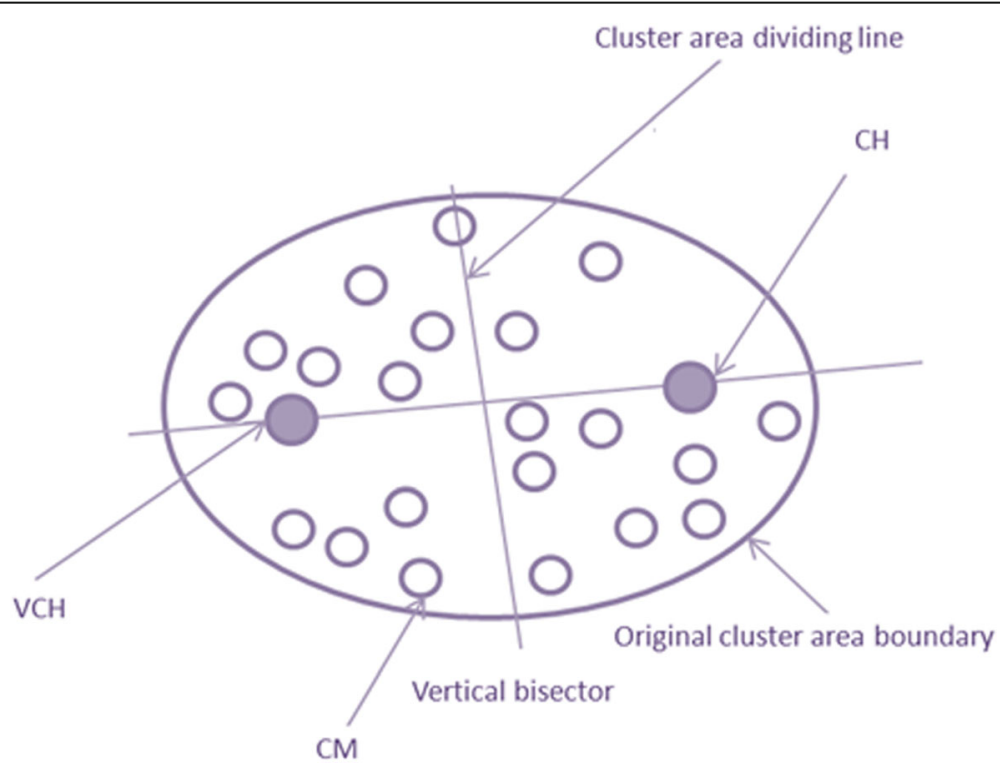

Fig. 2 Segmentation map of the cluster local area 
the formula for calculating the competition radius (boundary) of network nodes.

$$
R_{r}=\left\{1-c \frac{d_{\max }-d\left(s_{i}, \mathrm{BS}\right)}{d_{\max }-d_{\min }}\right\} R_{c \max }
$$

In formula (5), $g$ is the node competition radius, $c=d_{\min } /$ $d_{\max }, c \in(0,1)$ and $c$ is the degree of non-uniformity. That is, the smaller the value of $c$, the greater the difference between the two. $d_{\max }$ and $d_{\min }$ are the maximum and minimum values of the nodes to the BS in the WSN, respectively. The distance from node $s_{i}$ to the base station is $d\left(s_{i}, \mathrm{BS}\right)$, and $R_{c \max }$ is the maximum competition radius of the node set by the network. The above algorithm lacks the theoretical derivation of each hierarchical boundary (node competition radius). Therefore, a theoretical derivation method for obtaining the node competition radius by the energy consumption of each layer node is proposed. The energy consumption of each layer node is shown in Eq. (6).

$\left\{\begin{array}{c}E_{n}=\left(\pi \times r_{n}^{2} \times \rho\right) \times\left[E_{\text {elec }} \times k+\varepsilon_{f s} \times k \times\left(r_{n}+r_{n-1}\right)^{2}\right] \\ E_{n-1}=\left(\pi \times r_{n-1}^{2} \times \rho\right)+\left[\pi \times r_{n}^{2} \times \rho \times\left(\frac{L}{2 \times r_{n}}\right) \times\left(\frac{2 \times r_{n-1}}{L}\right)^{2}\right] \\ \times\left[E_{\text {elec }} \times k+\varepsilon_{f s} \times k \times\left(r_{n-1}+r_{n-2}\right)^{2}\right] \\ E_{1}=\left\{\pi \times r_{1}^{2} \times \rho+\left[\sum_{i=2}^{n} \pi \times r_{i}^{2} \times \rho \times\left(\frac{L}{2 \times r_{i}}\right) \times\left(\frac{2 \times r_{i}}{L}\right)\right]\right\} \\ \times\left[E_{\text {elec }} \times k+\varepsilon_{f s} \times k \times \bar{R}^{2}\right]\end{array}\right.$

In Eq. (6), $E_{i}$ is the total energy consumption of the $i$ th layer, $k$ is the data packet size sent by each node, and $\bar{R}$ is the average distance from the first layer to the sink node (base station). When $E_{n} \approx E_{n-1} \approx \cdots \approx E_{2} \approx E_{1}$, the competition radius of each layer node can be approximated, thus dividing the network. The closer the network layer is to the base station, the smaller the area of the layer, that is, the fewer the number of nodes, the less the local energy consumption, and communication resources are occupied, so that more energy and communication resources are used for relay transmission. After the network layering ends, the cluster head creates a LEMPT optimized path that passes through multiple hops $k$ to all cluster heads in the network.

Topology-Aware Resource Adaptation to Alleviate Congestion in Sensor Networks (TARA) is shown in Fig. 3

In the low-energy routing design, by controlling the minimum distance $r_{\min }$ between the cluster head nodes, the cluster head nodes can be evenly distributed in the network with the greatest possibility, and the variance of the cluster size is minimized. Therefore, the hot spot phenomenon in which cluster head nodes are too concentrated in Fig. 3a is unlikely to occur in the WSN network designed in this paper. Figure $3 \mathrm{~d}$ indicates that the cross-hotspots with overlapping traffic are unlikely to occur in more obvious application occasions of various areas. Figure $3 \mathrm{c}$ shows the cross-hotspot situation of traffic surge, which is mainly caused by the fact that the number of child nodes (neighbor nodes) of some nodes is large due to multi-hop within the cluster. Since the node bandwidth is narrow and the number of available channels with small interference is small, the amount of cached data of the node is large. It is highly likely to cause loss and congestion caused by packet overflow and packet wait timeout, which is explained and resolved. Figure $3 \mathrm{~b}$ indicates a hot spot situation in the base station (sink node) area. The main reason for this phenomenon is that the network cluster head node transmits data to the base station based on multi-hop multi-path transmission. The nodes near the base station have to bear the energy consumption and communication resources (channel allocation, occupation) pressure of data acquisition, processing, and transmission of nodes within the cluster. At the same time, it also bears the energy consumption of the relay transmission of almost all network data between other cluster heads and the base station and the load of the communication resources.

Figures 4 and 5 respectively show a schematic diagram of a communication model of a four-layer network and a twohop inter-cluster and a communication diagram of a fourlayer network and four-hop inter-cluster. The cluster head labeled by the triangle in the figure is the wake-up node. Some cluster head nodes need to accept and output more nodes' data. These nodes are consumed too much, and the link quality is degraded due to communication interference, which is likely to cause hot spot-congestion. To solve this problem, the base station becomes a cluster head by waking up the hot spot neighbor sleeping node, and the base station creates and updates the LEMPT path, and the newly added cluster head will be awakened and share part of the data traffic of the congested cluster head, which can largely alleviate or even cancel the network congestion.

\section{Network commodity selection system based on wireless sensor system}

On the server, the system deploys and adopts B/S three-tier architecture design, including data layer, functional layer, and presentation layer. Through layered design, each layer only needs to complete the functions of this layer and complete the functions provided for other layers, and the layers are independent of each other.

Figure 6 is a general structural diagram of the personalized commodity intelligent push system described by this paper.

\subsection{Presentation layer}

It provides an interface for users to interact with the system. Users can register, $\log$ in, browse services, 


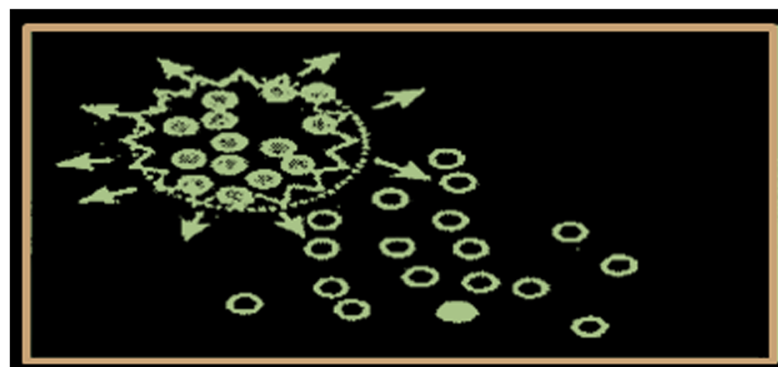

(a) Source hot sport

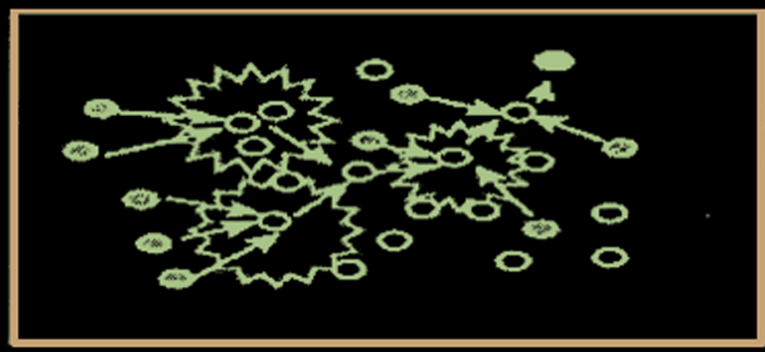

(c) Intersection hot sport-merging

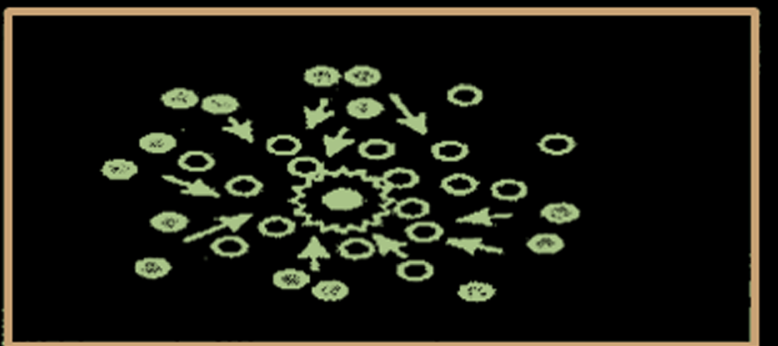

(b) Sink hot sport

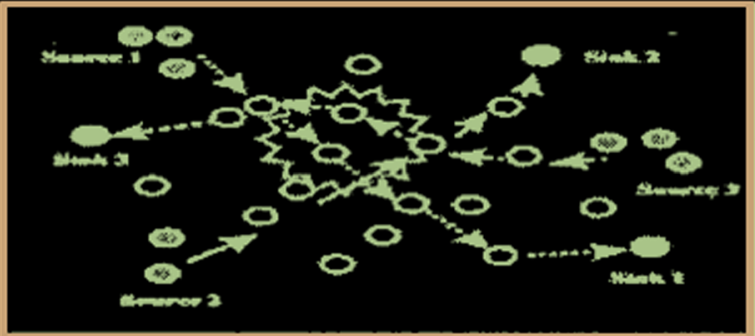

(d) Intersection hot spot-crossing traffic

Fig. 3 Three "hot spots" area scenarios

product information, view personal feature information, view push services, and product information on the browser page.

\subsection{Functional layer}

According to the user's personal information and collected feature data, the data mining related algorithm is used to process and recommend appropriate services and products to the user. After the user logs in to the system, the calculated recommendation result is pushed to the interface browsed by the user.

\subsection{Data layer}

The system data layer mainly stores data information in the system. The data of the system includes (1) data related to the user: user name, login password, personal information filled in, user personal health characteristic data collected by the wireless sensor network, and health

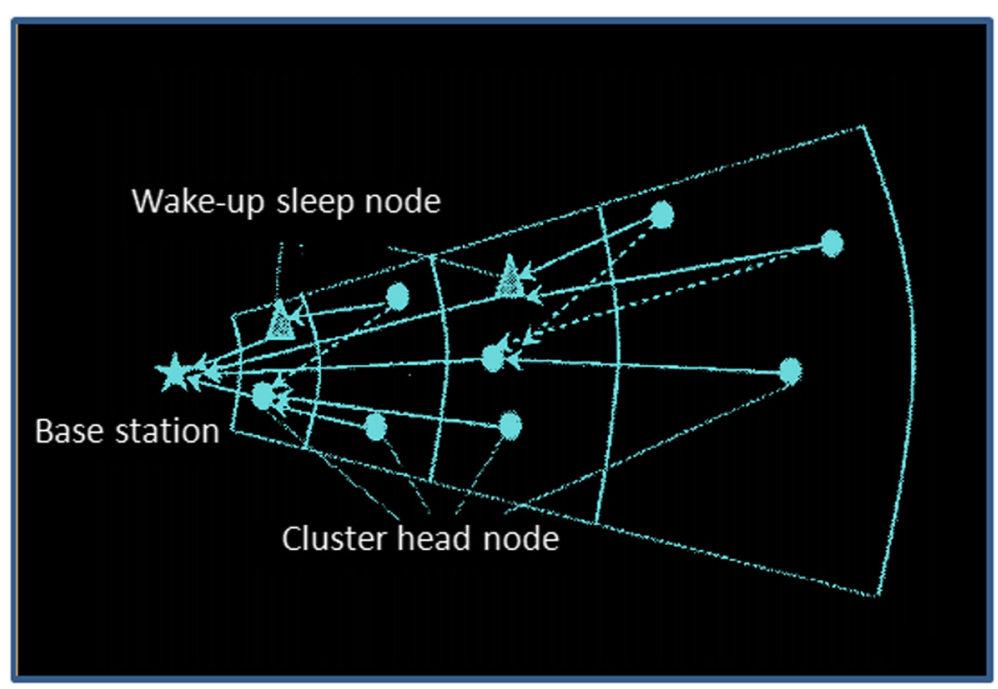

Fig. 4 Schematic diagram of intra-clusters multi-hop of four-layer and two-hop 


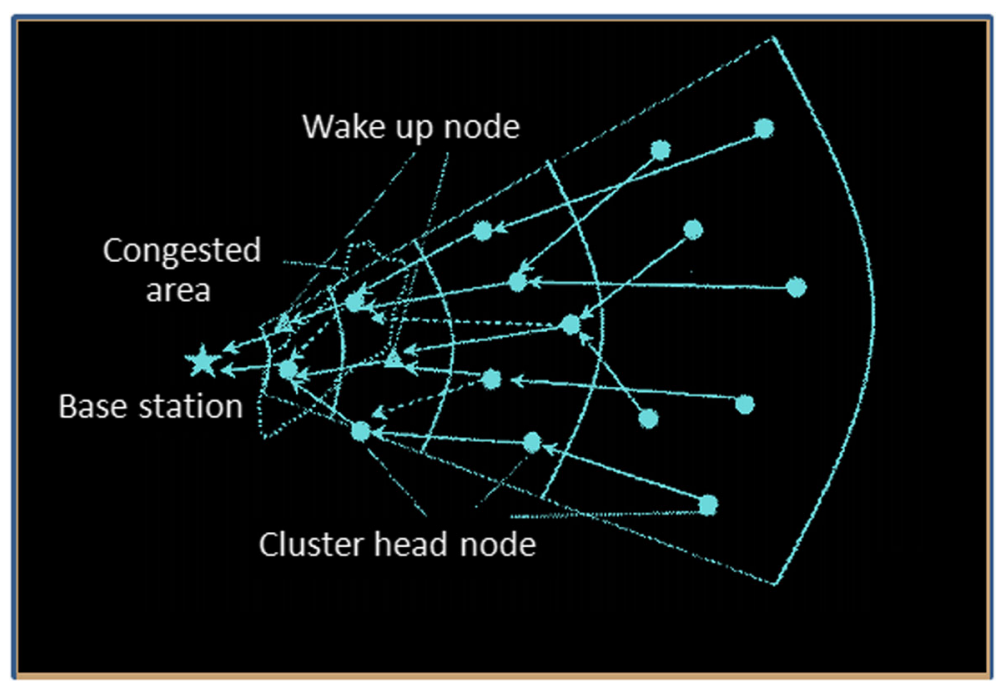

Fig. 5 Schematic diagram of multi-hop of four-layer and four-hop

service and product information previously selected by the user. (2) Information related to services and commodities. This type of information is mainly entered and maintained by the administrator in the background.

Figure 7 is a network architecture diagram of a consumer intelligent push system based on a wireless sensing system. The system mainly uses the idea of data mining to analyze and process the target user feature

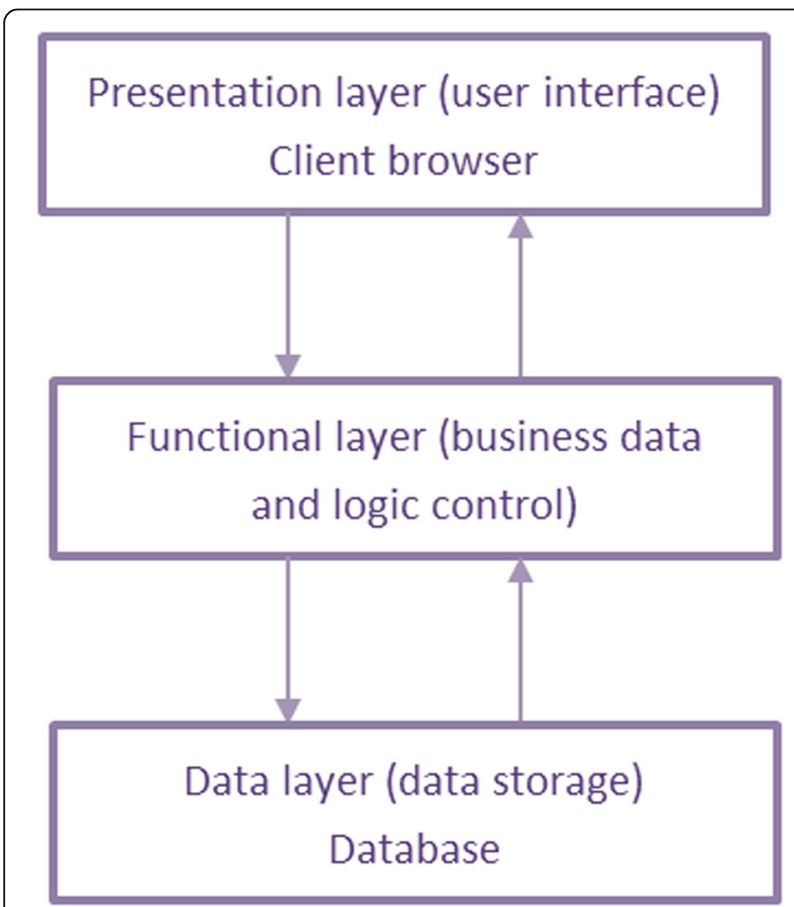

Fig. 6 Overall structure of the intelligent commodity push system data collected by the sensor terminal and obtain the set of neighboring users that are close to the target user status, and recommends the goods to the target users according to the products selected by other users in the past. Finally, the calculated and obtained recommendation results are actively pushed from the server to the user browsing interface.

\section{Analysis and discussion}

The quality of wireless sensor network communication services is mainly affected by network congestion. Researchers often use a variety of metrics such as cache queue length, cache rate, throughput, packet loss rate, network transmission delay, and network efficiency. However, these metrics are based on different application environments of wireless sensor networks. Therefore, there is always no single metric that can systematically and comprehensively evaluate the network congestion completely. In the general sense, network congestion means that the node accepts data packets faster than the sending rate, so that the data queue of the node buffer grows continuously. Network congestion will cause data throughput to drop, loss rate to increase, transmission delay to increase, network energy consumption to increase, and even more network failures and crashes.

Wireless sensor network congestion can seriously affect the reliability of network service quality (QOS) and data transmission, reduce energy utilization, and accelerate the decline of the network. Therefore, network congestion control strategy has become one of the key technologies in wireless sensor network design. While designing the network congestion algorithm, we must also consider the network transmission quality, dynamic adaptability, robustness, energy utilization, timely prediction, network 


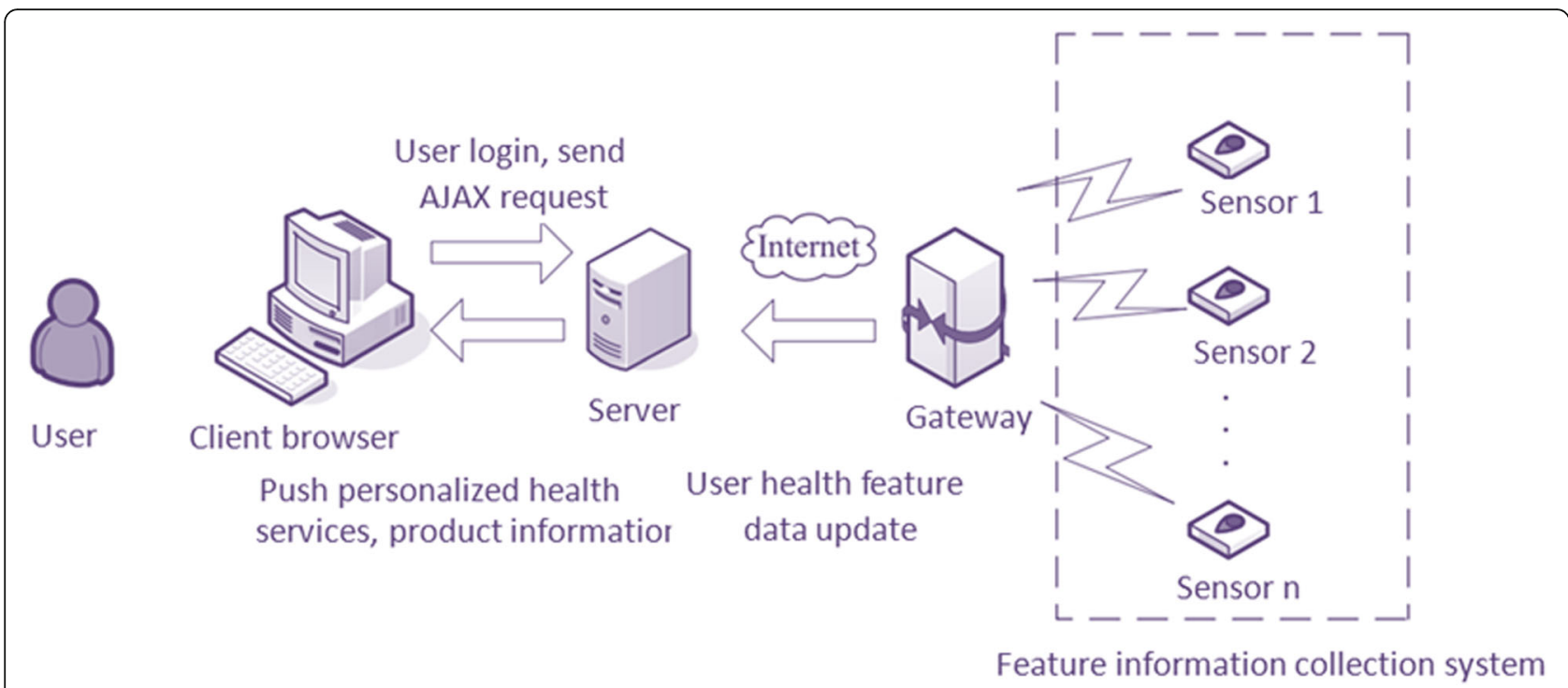

Fig. 7 Network architecture diagram of a consumer intelligent push system based on a wireless sensing system

congestion avoidance, and cancellation in different application scenarios. Each type of congestion control strategy uses congestion detection to achieve network congestion prediction and timely discovery, and further achieves network congestion mitigation through congestion avoidance. Finally, network congestion or local congestion elimination is achieved through data transmission mode adjustment during congestion cancellation.

\subsection{Congestion detection}

In different application scenarios of the WSN, the congestion prediction of the network congestion is often implemented by monitoring parameters such as the length and rate of the cache queue, the time at which the node CPU processes the data packet, the ratio of the data packet input rate, and the ratio of the packet processing or forwarding rate. In addition, congestion detection can be realized by monitoring parameters such as increased network packet loss rate, network delay time increase, channel communication load increase, or interference enhancement.

\subsection{Congestion avoidance}

After the WSN is discovered, the congestion control data packet is sent to the upstream node or directly to the node where the entire network is congested. The network has the following two types of congestion feedback: one is that the sensor network generates congestion control packets and is sent directly to the congestion source node, and the other is that the sensor network uses the control packet or the congestion control bit in the data packet and feeds back it to the congestion source node. After that, the congestion source node judges the congestion state by receiving different data packets or control packets, and then executes the corresponding congestion control strategy.

\subsection{Congestion lifted}

The upstream node that receives the congestion packet uses a series of congestion control methods to implement congestion mitigation and release. In order to eliminate the congestion caused by the wireless link layer, WSN mostly adopts strategies such as optimizing the topology and introducing a wireless channel allocation protocol such as CSMA/CA. For node-level network congestion, the congestion control strategy basically adopts methods such as reducing the data transmission rate or dropping the congestion data packet. In addition, some congestion control strategies are designed to achieve congestion mitigation and release by designing corresponding routing algorithms.

\section{Conclusion}

Wireless sensors are used to collect product information viewed by consumers. After late data processing and data mining, more potential information can be obtained, such as color preference and commodity turnover rate. This information can provide data support, quantitative analysis, and effective estimates for the merchants to do the next marketing plan and strategy, store layout planning, and other initiatives. Moreover, this paper focuses on the wireless sensor network data acquisition system in the network commodity sales system and combines the operating conditions of the network commodity store to develop wireless sensor network hardware design, routing design, network congestion control strategy, database design, and construction. In addition, the system mainly uses the idea of data mining 
to analyze and process the target user feature data collected by the sensor terminal and obtain a set of neighboring users close to the target user. After that, the target user is recommended according to the previously selected product status of other users in the neighborhood user group. Finally, the calculated recommendation results are actively pushed from the server to the user browsing interface.

\section{Acknowledgements}

The authors gratefully acknowledge the helpful comments and suggestions of the reviewers, which have improved the presentation.

\section{Authors' contributions}

All authors take part in the discussion of the work described in this paper.

\section{Funding}

This work was supported by Humanity and Social Science foundation of MOE of China (No.20171304).

\section{Availability of data and materials}

Please contact the corresponding author for data requests.

\section{Competing interests}

These no potential competing interests in our paper.

Received: 30 May 2019 Accepted: 20 August 2019

Published online: 02 September 2019

\section{References}

1. A. Hentati, E. Driouch, J.F. Frigon, et al., Fair and low complexity node selection in energy harvesting wireless sensor networks [J]. IEEE Systems Journal 2017, 1-11

2. A. Al-Baz, A. El-Sayed, A new algorithm for cluster head selection in LEACH protocol for wireless sensor networks [J]. International Journal of Communication Systems 31(1), 01-13 (2018)

3. F. Haleem, J. Huma, J. Bilal, et al., Analytical network process based optimum cluster head selection in wireless sensor network [J]. PLOS ONE 12(7), e0180848 (2017)

4. K. Nitesh, M. Azharuddin, P. Jana, Minimum spanning tree-based delayaware mobile sink traversal in wireless sensor networks: delay-aware mobile sink traversal in WSN [J]. International Journal of Communication Systems 30(13), e3270 (2017)

5. A. Keshavarz-Mohammadiyan, H. Khaloozadeh, Interacting multiple model and sensor selection algorithms for manoeuvring target tracking in wireless sensor networks with multiplicative noise [J]. International Journal of Systems Science 48(5), 10 (2017)

6. M. Taherian, M. Maeen, M. Haghparast, Promoting the quality level of signaling in railway transportation system taking advantage from wireless sensor networks technology [J]. Computers 6(3), 26 (2017)

7. Y.C. Chou, M. Nakajima, A clonal selection algorithm for energy-efficient mobile agent itinerary planning in wireless sensor networks [J]. Mobile Networks and Applications (2017)

8. V. Talla, M. Hessar, B. Kellogg, et al., LoRa Backscatter [J]. Proceedings of the ACM on interactive, mobile, wearable and ubiquitous technologies 1(3), 124 (2017)

9. S.D. Trapasiya, H.B. Soni, Energy efficient policy selection in wireless sensor network using cross layer approach [J]. IET Wireless Sensor Systems 7(6), 191-197 (2017)

10. H. Zhu, F. Xiao, L. Sun, et al., R-TTWD: Robust device-free through-the-wall detection of moving human with WiFi [J]. IEEE Journal on Selected Areas in Communications, 1-1 (2017)

11. GPU Parallel Implementation of Spatially Adaptive Hyperspectral Image Classification [J]. IEEE Journal of Selected Topics in Applied Earth Observations \& Remote Sensing, 2017, PP(99):1-13.

12. M. Hosseini, H. Mcnairn, Using multi-polarization C- and L-band synthetic aperture radar to estimate biomass and soil moisture of wheat fields [J]. International Journal of Applied Earth Observation and Geoinformation 58, 50-64 (2017)
13. J. Yang, H. Zou, H. Jiang, et al., CareFi: sedentary behavior monitoring system via commodity WiFi infrastructures [J]. IEEE Transactions on Vehicular Technology 67(8), 7620-7629 (2018)

14. X. Zheng, J. Wang, L. Shangguan, et al., Design and implementation of a CSI-based ubiquitous smoking detection system [J]. IEEE/ACM Transactions on Networking 25(6), 3781-3793 (2017)

15. H. Deng, J. Wu, L. Zhu, et al., Texture edge-guided depth recovery for structured light-based depth sensor [J]. Multimedia Tools and Applications 76(3), 4211-4226 (2017)

16. Y. Lu, J. Zhang, B. Li, et al., Harnessing commodity wearable devices to capture learner engagement [J]. IEEE Access, 99):1-99):1 (2019, PP)

17. G. Yu, Z. Yifan, L. Jie, et al., Sleepy: wireless channel data driven sleep monitoring via commodity WiFi devices [J]. IEEE Transactions on Big Data, 1-1 (2018)

18. R. Zhang, N. Zhang, C. Du, et al., From electromyogram to password [J]. ACM Transactions on Intelligent Systems and Technology 9, 1):1-1)20 (2017)

19. M. Karar, S. Paul, A. Mallick, et al., Interaction behavior between active hydrogen bond donor-acceptors as a binding decoration for anion recognition: experimental observation and theoretical validation [J]. ChemistrySelect 2(9), 2815-2821 (2017)

20. H. Rueda, C. Fu, D.L. Lau, et al., Single aperture spectral+ToF compressive camera: toward hyperspectral+depth imagery [J]. IEEE Journal of Selected Topics in Signal Processing 11(7), 992-1003 (2017)

\section{Publisher's Note}

Springer Nature remains neutral with regard to jurisdictional claims in published maps and institutional affiliations.

\section{Submit your manuscript to a SpringerOpen ${ }^{\circ}$ journal and benefit from:}

- Convenient online submission

- Rigorous peer review

- Open access: articles freely available online

- High visibility within the field

- Retaining the copyright to your article

Submit your next manuscript at $\boldsymbol{\nabla}$ springeropen.com 